\title{
Energy Conservation Law in the Closed Universe and a Concept of the Proper Time
}

\author{
Natalia Gorobey, Alexander Lukyanenko * and Pavel Drozdov
}

Peter the Great Saint Petersburg Polytechnic University, Polytekhnicheskaya 29, 195251 St. Petersburg, Russia; n.gorobey@mail.ru (N.G.); drozdov.pv@edu.spbstu.ru (P.D.)

* Correspondence: alex.lukyan@mail.ru

Received: 12 September 2020; Accepted: 8 October 2020; Published: 12 October 2020

\begin{abstract}
To define time in the homogeneous anisotropic Bianchi-IX model of the universe, we propose a classical equation of motion of the proper time of the universe as an additional gauge condition. This equation is the law of conservation of energy. As a result, a new parameter, called a "mass" of the universe, appears. This parameter is added to the anisotropy energy and regarded as an observed quantity. The "mass" of the universe is decisive when it comes to the dynamics of its origin.
\end{abstract}

Keywords: anisotropic universe; quantum theory; Hamiltonian; constraint equations; proper time; mass of the universe

\section{Introduction}

The ordinary approach to the quantization of covariant theories is based on the expansion of the phase space by adding Lagrange multipliers, the corresponding canonical momenta, and ghosts [1-4]. In the case of a relativistic particle, the covariant functional integral includes one additional integration over proper time [5]. This integral reduces to representing the Green functions for the Dirac and Klein-Gordon equations proposed by Fock [6] and Schwinger [7]. In [8], a "shortened" version of covariant quantum theory was formulated with minimal expansion of the phase space, including parameters of finite transformations of the covariance group. These parameters include the proper time. However, integration over these parameters deprives the functional integral of dynamic meaning. This means that the gauge conditions, which are assumed, in particular, in [5], are not sufficient for defining the time parameter. In [9], an additional expansion of the phase space was proposed by including infinitesimal shifts of new variables (and corresponding momenta) there. This means that the law of conservation of the universe's energy is an additional condition for time defining. An additional condition for the action of general relativity is also added in papers $[10,11]$ to introduce a special type of scalar field. In our case, the modification plays the role of a gauge condition that violates the covariance. The consequences of this modification of the classical general relativity are considered in this work for the homogeneous anisotropic model of the Bianchi-IX universe [12]. A complete set of generalized coordinates of a modified theory, in addition to proper time, will include its energy, which is a generator of infinitesimal shifts. This value is an integral of motion and is included in the set of model parameters.

\section{Modification of a Homogeneous Anisotropic Model of the Universe}

We take the Lagrange function of the original model in the form (hereinafter, we assume the speed of light $c=1$ )

$$
L=\frac{1}{2 g}\left[\left(\frac{a \dot{a}^{2}}{N}-a N\right)-\left(a^{3} \frac{\dot{\beta}_{+}^{2}+\dot{\beta}_{-}^{2}}{N}-a N V\left(\beta_{+}, \beta_{-}\right)\right)\right]+\Lambda a^{3} \cdot N,
$$


where $g=2 G / 3 \pi$ and

$$
V\left(\beta_{-}, \beta_{+}\right)=\frac{1}{3} e^{-8 \beta_{+}}-\frac{4}{3} e^{-2 \beta_{+}}+\cosh 2 \sqrt{3} \beta_{-}+1+\frac{2}{3} e^{4 \beta_{+}}\left(\cosh 4 \sqrt{3} \beta_{-}-1\right) .
$$

Here, $N(\tau)$ is an arbitrary function of the parameter $\tau$ (lapse function) [12]. We will carry out both stages of the modification immediately. At the first stage, we introduce our proper time $s$, according to

$$
N=\dot{s}
$$

Given the fact that $N$ is an arbitrary function, introducing a new function $s$ does not contradict anything. The modification occurs when we assume that $s$ is a dynamical variable. In fact, it occurs by introducing the proper time as a dynamical variable in relativistic mechanics [6]. We find the Euler-Lagrange equation for $s$, and subsequently add it to the initial action, multiplying by the corresponding Lagrange multiplier. Obviously, this step does not change the dynamical aspect of classical theory. The procedure mentioned above is equivalent to the addition of variation in the initial Lagrangian, generated by an infinitesimal shift in the proper time

$$
\delta s=-\epsilon,
$$

to this Lagrangian. This fact is sufficient for (according to [8,9]) removing additional integration over the proper time in the quantum theory. Here, we consider the consequences of the proposed modification, which appears in the classical theory. As a result, two new dynamic variables $s, \epsilon$ appear, and the modified Lagrange function has the form

$$
\tilde{L}=\frac{1}{2 g}\left[\frac{a \dot{a}^{2}}{\dot{s}}\left(1+\frac{\dot{\epsilon}}{\dot{s}}\right)-a(\dot{s}-\dot{\epsilon})\right]-\frac{1}{2 g}\left[a^{3} \frac{\dot{\beta}_{+}^{2}+\dot{\beta}_{-}^{2}}{\dot{s}}\left(1+\frac{\dot{\epsilon}}{\dot{s}}\right)-a \cdot(\dot{s}-\dot{\epsilon}) V\right]+(\dot{s}-\dot{\epsilon}) \Lambda a^{3} .
$$

We turn to the canonical form of the modified theory. Find the canonical momenta

$$
\begin{gathered}
p_{a}=\frac{1}{g} \frac{a \dot{a}}{\dot{s}}\left(1+\frac{\dot{\epsilon}}{\dot{s}}\right), \\
p_{ \pm}=-\frac{a^{3}}{g} \frac{\dot{\beta}_{ \pm}}{\dot{s}}\left(1+\frac{\dot{\epsilon}}{\dot{s}}\right), \\
p_{s}=-\frac{1}{2 g}\left[\frac{a \dot{a}^{2}}{\dot{s}^{2}}\left(1+2 \frac{\dot{\epsilon}}{\dot{s}}\right)+a\right]+\frac{1}{2 g}\left[a^{3} \frac{\dot{\beta}_{+}^{2}+\dot{\beta}_{-}^{2}}{\dot{s}^{2}}\left(1+2 \frac{\dot{\epsilon}}{\dot{s}}\right)+a V\right]+\Lambda a^{3}, \\
P_{\epsilon}=\frac{1}{2 g}\left(\frac{a \dot{a}^{2}}{\dot{s}^{2}}+a\right)-\frac{1}{2 g}\left(a^{3} \frac{\dot{\beta}_{+}^{2}+\dot{\beta}_{-}^{2}}{\dot{s}^{2}}+a V\right)-\Lambda a^{3} .
\end{gathered}
$$

It is easy to verify that the modified Hamiltonian is also equal to zero, as it should be for the reparameterization-invariant theory. Moreover, Equation (8) gives the Hamiltonian constraint

$$
\begin{array}{r}
\tilde{H}=p_{s}-2 \sqrt{P_{\epsilon}-\frac{a}{2 g}+\frac{a V}{2 g}+\Lambda a^{3}} \sqrt{\frac{g p_{a}^{2}}{2 a}-\frac{g\left(p_{+}^{2}+p_{-}^{2}\right)}{2 a^{3}}} \\
+P_{\epsilon}-2\left(\frac{a}{2 g}-\frac{a V}{2 g}\right)+\Lambda a^{3}=0 .
\end{array}
$$

We write the modified action in canonical form as follows

$$
\tilde{I}=\int_{0}^{1} d \tau\left(p_{a} \dot{a}+p_{+} \dot{\beta}_{+}+p_{-} \dot{\beta}_{-}+p_{s} \dot{s}-\epsilon \dot{P}_{\epsilon}-\tilde{N} \tilde{H}\right) .
$$


Now, we consider an infinitesimal shift $\epsilon$ as a canonical momentum.

As follows from Equation (11), the additional integral over $\epsilon$ in quantum theory gives a functional $\delta$-function

$$
\prod_{\tau} \delta\left(\dot{P}_{\epsilon}\right)
$$

from which we obtain $P_{\epsilon}$ as the constant of motion.

\section{3. "Mass" of the Universe}

The appearance of the integral of motion $P_{\epsilon}$ and its meaning will be explained by the example of a relativistic particle. Initially, we take the case of a massless particle with the Lagrange function

$$
L_{m}=\frac{1}{2} \frac{\dot{x}_{\mu} \dot{x}^{\mu}}{N}
$$

Bearing in mind (3), we add the Lagrangian variation generated by the infinitesimal shift in the proper time (4)

$$
\tilde{L}_{m}=\frac{1}{2} \frac{\dot{x}_{\mu} \dot{x}^{\mu}}{N}\left(1+\frac{\dot{\epsilon}}{N}\right) .
$$

After this modification, the Hamiltonian constraint takes the form

$$
\tilde{H}_{m}=\sqrt{2 P_{\epsilon}} \sqrt{p_{\mu} p^{\mu}}-P_{\epsilon} .
$$

The constraint is the square root of the Hamiltonian constraint of a relativistic particle with mass $m$, if we put

$$
P_{\epsilon}=2 m^{2} \text {. }
$$

Thus, as a result of this modification, the particle "gained" mass. We will also define "mass" of the universe by the Formula (16).

If we assume the canonical gauge condition $\dot{s}=\tilde{N}$, the modified action (11) takes the form

$$
\tilde{I}=\int_{0}^{1} d \tau\left(p_{a} \dot{a}+p_{+} \dot{\beta}_{+}+p_{-} \dot{\beta}_{-}-\tilde{N} \tilde{h}\right)
$$

where

$$
\tilde{h}=-2 \sqrt{2 m^{2}-\frac{a}{2 g}+\frac{a V}{2 g}+\Lambda a^{3}} \sqrt{\frac{g p_{a}^{2}}{2 a}-\frac{g\left(p_{+}^{2}+p_{-}^{2}\right)}{2 a^{3}}}+2 m^{2}-\frac{a}{g}+\frac{a V}{g}+\Lambda a^{3}=0 .
$$

If we put, as is commonly believed, the "mass" of the universe equal to zero, then it is easy to see that, as a result, we obtain the square root of the Hamiltonian constraint of the original theory. Here, we assume that this mass is nonzero and see what this leads to in classical theory. Put, as usual, in this case, $\tilde{N}=1$. Then, one of the equations of motion will be

$$
\frac{1}{2 g} a\left(\dot{a}^{2}+1\right)=2 m^{2}+\Lambda a^{3}+\frac{1}{2 g}\left[a^{3}\left(\dot{\beta}_{+}^{2}+\dot{\beta}_{-}^{2}\right)+a V\right],
$$

In the absence of anisotropy for the simplest homogeneous model of the universe, Equation (19) takes the form

$$
\frac{1}{2 g} a\left(\dot{a}^{2}+1\right)=2 m^{2}+\Lambda a^{3}
$$

Its solution at $\Lambda=0$ has the form

$$
\arcsin \frac{\sqrt{a}}{2 m \sqrt{g}}-\frac{\sqrt{a}}{2 m \sqrt{g}} \sqrt{1-\frac{a}{4 g m^{2}}}=\frac{s}{4 g m^{2}} .
$$


and the general solution has such an asymptotic behavior near $a=0$. We see, therefore, that the evolution of the universe near its origin is determined by its "mass". At all stages of the evolution of the universe, its "mass" is present as an additive to the energy of matter. The solutions of the Equation (21) for different $\Lambda$ are represented on the graph. The red dashed line on the Figure 1 corresponds to the solution in the case $\Lambda=0$.

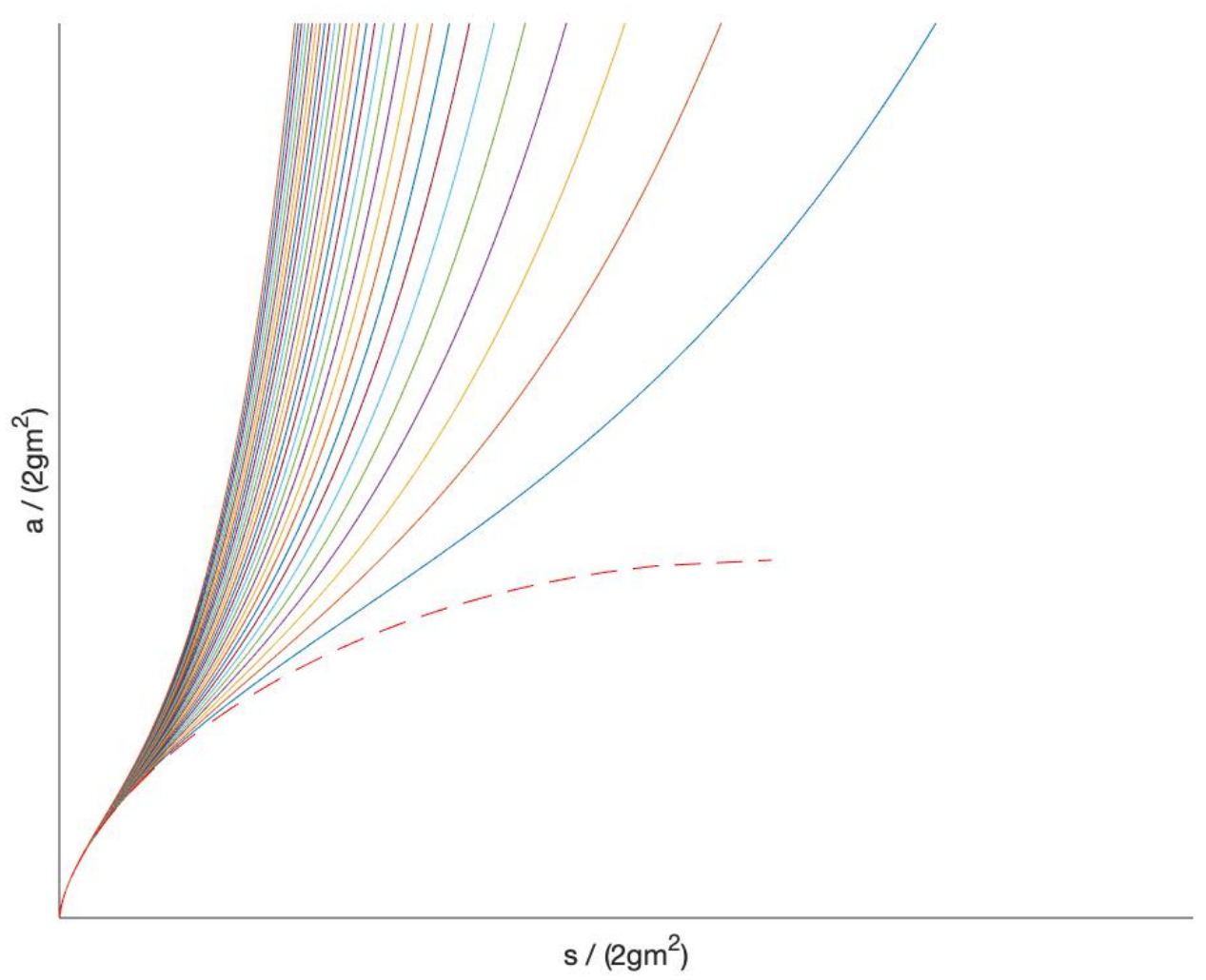

Figure 1. The graph of the solutions determined.

\section{Conclusions}

The modification to the dynamics of the universe suggested in this article is not arbitrary. It is one of the possible options for a solution to the problem of time in covariant quantum theory. However, consequently, it causes the advent of an additional parameter, called a "mass" of the universe in classical theory. This quantity is not connected to the energy of a common matter (in this case, it is anisotropic energy) but added to it for the balance of energy in the closed universe. This additive is determinative near the origin of the universe, allowing it to alleviate the singularity problem. Therefore, it is observable in principle. At the next stages of the universe evolution, the proper mass exists in the form of the "dark" contribution to its dynamics. If we assume the "mass" of the universe equal to zero, the modified theory will reduce to the source theory, with necessary extraction of the square root of the Hamiltonian constraint.

Author Contributions: Conceptualization, N.G. and A.L.; methodology, A.L.; software, P.D.; validation, N.G., A.L. and P.D.; formal analysis, A.L.; investigation, A.L., P.D.; resources, A.L.; data curation, A.L.; writing-original draft preparation, N.G., A.L.; writing-review and editing, A.L., P.D.; visualization, P.D.; supervision, A.L.; project administration, A.L. All authors have read and agreed to the published version of the manuscript.

Funding: This research received no external funding.

Acknowledgments: The authors thank V.A. Franke and A.V. Goltsev for useful discussions.

Conflicts of Interest: The authors declare no conflict of interest. 


\section{References}

1. Fradkin, E.S.; Vilkovisky, G.A. Quantization of relativistic systems with constraints. Phys. Lett. 1975, 55, 224-226. [CrossRef]

2. Batalin, I.A.; Vilkovisky, G.A. Relativistic S-matrix of dynamical systems with boson and fermion constraints. Phys. Lett. 1975, 69, 309-312. [CrossRef]

3. Henneaux, M. Hamiltonian form of the path integral for theories with a gauge freedom. Phys. Rep. 1985, 126, 1-66. [CrossRef]

4. Ore, F.R.; van Nieuwenhuizen, P. Local BRST symmetry and the geometry of gauge-fixing. Nucl. Phys. B 1982, 204, 317-332. [CrossRef]

5. Govaerts, J. A note of the Fradkin-Vilkovisky theorem. Int. J. Mod. Phys. A 1988, 4, 4487-4504. [CrossRef]

6. Fock, V.A. Die Eigenzeit in der klassischen- und in der Quanten-mechanik. Bull. Russ. Acad. Sci. 1937, 2, 568-576.

7. Schwinger, J. On gauge invariance and vacuum polarization. Phys. Rev. D 1951, 82, 664-679. [CrossRef]

8. Gorobey, N.; Lukyanenko, A. Time and Observables in Covariant Quantum Theory. arXiv 2020, arXiv:2001.09003v1.

9. Gorobey, N.; Lukyanenko, A.; Goltsev, A.V. Feynman Propagator for a System of Interacting Scalar Particles in the Fokker Theory. arXiv 2020, arXiv:2002.03607v1.

10. Lim, E.A.; Sawicki, I.; Vikman, A. Dust of dark energy. J. Cosmol. Astropart. Phys. 2010, 2010, 12. [CrossRef]

11. Chamseddine, A.H.; Mukhanov, V. Mimetic dark matter. J. High Energ. Phys. 2013, 2013, 135. [CrossRef]

12. Misner, C.W.; Thorne, K.S.; Wheeler, J.A. Gravitation; W.H. Freeman and Company: San Francisco, CA, USA, 1973; pp. 487-500.

(C) 2020 by the authors. Licensee MDPI, Basel, Switzerland. This article is an open access article distributed under the terms and conditions of the Creative Commons Attribution (CC BY) license (http:/ / creativecommons.org/licenses/by/4.0/). 